\title{
Gastrodin ameliorates spinal cord injury via antioxidant and anti-inflammatory effects
}

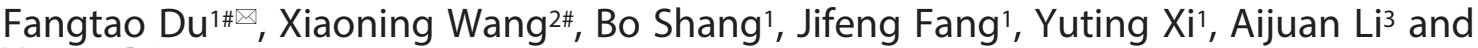 \\ Yenze Diao ${ }^{4}$
}

'Department of Traumatic Orthopedics, The Second People's Hospital of Liaocheng, TaiShan Medical College, Liaocheng 252600, China; ${ }^{2}$ Department of Orthopedics, The People's Hospital of Gaotang, Liaocheng 252800, China; 'Bepartment of Outpatient, The Sixth People's Hospital of Weifang, Weifang 261021, China; ${ }^{4}$ Department of Orthopedics, The Peking University Third Hospital, Beijing 100191, China

\begin{abstract}
Spinal cord injury ( $\mathrm{SCl}$ ) is one of the most severe traumatic injuries that results in dysfunction of limbs and trunk below the damaged section. Recent studies have shown that gastrodin (GAS) could improve the recovery of SCl. In the current study, we aimed to examine the possible mechanism underlying the effect of GAS on recovery of $\mathrm{SCl}$ in rats. In rats with $\mathrm{SCl}$, GAS improved locomotor functions and decreased permeability of blood-spinal cord barrier, as illustrated by increase of Basso-Beattie-Bresnahan scores and decrease of Evans blue leakage. In addition, GAS inhibited inflammation, as evidenced by decrease of proinflammatory cytokines, including tumor necrosis factor a (TNFa) and interleukin-1 $\beta$ (IL-1 $\beta$ ) in rats following SCI. Moreover, increase of TBARS content and decrease of glutathione (GSH) content and superoxide dismutase (SOD) activities in SCl rats were inhibited by GAS. Furthermore, GAS enhanced mRNA expression of nuclear factor (erythroid-derived 2)like 2 (Nrf2), catalytic subunit of $\gamma$-glutamylcysteine ligase $(G C L c)$ and modified subunit of $\gamma$-glutamylcysteine ligase (GCLm). The data suggested that GAS may promote the recovery of $\mathrm{SCl}$ through the enhancement of Nrf2-GCLC/ GCLm signaling pathway, and subsequent improvement of oxidative stress and inflammation, resulting in decrease of permeability of BSCB and improved recovery of locomotor function in rats with $\mathrm{SCl}$. The results have provided novel insights into GAS-related therapy of $\mathrm{SCl}$ and associated neurodegenerative diseases.
\end{abstract}

Key words: spinal cord injury; gastrodin; inflammation; oxidative stress; Nrf2

Received: 12 February, 2016; revised: 17 March, 2016; accepted: 19 May, 2016; available on-line: 30 July, 2016

\section{INTRODUCTION}

Spinal cord injury (SCI) is one of the most severe traumatic injuries that results in dysfunction of limbs and trunk below the damaged section, leading to postoperative complications, including spinal cord edema and cell death in the injured areas, and even permanent disability and death (Giuliano et al., 1999; Edwards et al., 2014). Due to the progress of several advanced techniques, including immunotherapy, tissue transplantation, and other advanced reconstruction methods, the treatment of SCI has been substantially developed. However, the severe damage induced by SCI significantly impaired the life quality of patients. SCI includes acute and secondary injuries. The acute SCI could result in a bruise, a partial tear, or a complete tear in the spinal cord (Alkabie \&
Boileau, 2015; Sothmann et al., 2015). The primary SCI could degrade key cytoskeletal and activate functional proteins, leading to delayed death of local and adjacent neurons and glial cells (Dionyssiotis et al., 2014; Henke et al., 2015). Primary injury is considered to be an irreversible mechanical damage (Evaniew et al., 2015). In contrast, the secondary injury is reversible which could be altered by potential interventions (Chen et al., 2015). Therefore, large amount of studies have focused on the understanding of the mechanism of SCI-related secondary injury and developing more efficient therapeutic options that can ameliorate and even reverse secondary damage of SCI.

The phenolic glucoside gastrodin (GAS) is a main phenolic compound and an active component derived from tall gastrodia tuber (Park et al., 2011). It is wellknown that GAS could improve the elasticity of largeand medium-sized artery walls, expand cerebral vessels, increase blood supply, relieve pain, and impart resistance to convulsions (Song et al., 2013). Literature in recent years has shown that GAS exhibits potent neuroprotective effects. Xu and coworkers (2007) reported that GAS had a neuroprotective action against hypoxia in cultured cortical neuron. Dai and coworkers (2011) found that GAS inhibited the expression of inducible NO synthase, cyclooxygenase- 2 and proinflammatory cytokines in cultured LPS-stimulated microglia via MAPK pathways. Sunand coworkers (2004) reported that GAS exerted neuroprotective effects by suppressing excitotoxicity, inhibiting free radical injury, resisting impairments in energy metabolism and suppressing apoptosis. GAS may also improve learning ability and facilitate memory consolidation and retrieval. Recently, it was found that GAS promoted the secretion of brain-derived neurotrophic factor, contributed to the recovery of neurological function, and protected neural cells after SCI (Song et al., 2013). However, the mechanism underlying the protective effect of GAS on the recovery of SCI are largely unknown.

In the present study, we aimed to investigate the effect of GAS on SCI and to elucidate the possible mechanism. The results showed that GAS may pro-

e-mail: fangtao_du999@126.com

\#They contributed equally to this paper

Abbreviations: BBB, Basso-Beattie-Bresnahan; BSCB, blood-spinal cord barrier; EB, Evans blue; GAS, gastrodin; GCLC, catalytic subunit of $\gamma$-glutamylcysteine ligase; GCLm, modified subunit of $\gamma$-glutamylcysteine ligase; GSH, glutathione; IL-1 $\beta$, interleukin-1 $\beta$; Nrf2, nuclear factor (erythroid-derived 2)-like 2; ROS, reactive oxygen species; $\mathrm{SCl}$, Spinal cord injury; SOD, superoxide dismutase; TBARS, TBA reactive substances; TNFa, tumor necrosis factor $a$. 
mote the recovery of SCI through the enhancement of Nrf2-GCLc/GCLm signaling pathway, and then improvement of oxidative stress and inflammation, resulting in decreased permeability of BSCB and improved recovery of locomotor function in rats with SCI.

\section{MATERIALS AND METHODS}

Animal treatment. The adult male Sprang-Dawley rats weighing 250-300 $\mathrm{g}$ were purchased from the Experimental Animals Center of TaiShan Medical College. All animal experiments were conducted in accordance with the National Institute of Health Guide on the Care and Use of Laboratory Animals and were approved by the Laboratory Animal Users Committee at TaiShan Medical College. All animals were housed in individual cages in a temperature and light \pm dark cycle controlled environment with free access to food and water.

The operation was conducted as previously reported (Hu et al., 2013; Song et al., 2013). The animals were anesthetized with an intraperitoneal injection of $10 \%$ chloral hydrate $(3 \mathrm{mg} / \mathrm{kg})$. The skin above the vertebral column was shaved, and then a $15-\mathrm{mm}$ midline skin was cut and dorsal muscles were separated to the side to expose the thoracic region of vertebral column (T8-T13). 60 rats were randomly and blindly divided into four groups with 15 rats in each group, including sham-operated (Sham) group, SCI group, SCI + low dose of GAS group (SCI + L-GAS) and SCI + high dose of GAS group (SCI + H-GAS). In Sham group, rats were subjected to the surgical procedure without the SCI operation; In SCI group, rats were subjected to SCI and intraperitoneally (i.p.) treated with vehicle; In SCI + GAS groups, rats subjected to SCI and i.p. injected with GAS (100 or 200 $\mathrm{mg} / \mathrm{kg}$ ) (TAUTO BIOTECH, China), every $24 \mathrm{~h}$ for 5 days, starting half an hour after SCI.

Behavioral Assessment. Locomotor activity was evaluated prior to surgery and at 1, 3, 7, 14, 21 and 28 days post-injury in all groups, based on a 21-point Basso-Beattie-Bresnahan (BBB) Locomotor Rating Scale, where 0 reflects no locomotion and 21 reflects normal motor functions (Basso et al., 1995; Hu et al., 2015). The experiment and calculation of scores of locomotor function were conducted by two independent and welltrained investigators according to the $\mathrm{BBB}$ scales in a blinded manner. Two independent examiners who were blinded to the experimental design observed hind limb movements, trunk position and stability, tail position, stepping, coordination, paw placement and toe stretching over a $5 \mathrm{~min}$ period for each rat (Hu et al., 2015).

Measurement of blood-spinal cord barrier (BSCB) permeability. Evans blue (EB) was used as a marker of albumin extravasation and EB leakage was detected to evaluate BSCB permeability. EB injection and sample collection were conducted as previously reported (Yu et al., 2014). Results were expressed as $\mu \mathrm{g} \mathrm{EB/g}$ spinal cord tissue.

Real-time PCR. Total RNA was extracted using the Trizol method, according to the manufacturer's instructions (Tiangen, China). After reverse transcription of cDNA, quantitative real-time PCR (qRT-PCR) was analyzed in a BIORAD CFX96 Real-Time System, using SYBR Premix Ex TaqTM II (Tiangen, China). The expression of genes was normalized to $\beta$-actin and comparatively analyzed using the $2^{-\triangle \Delta \mathrm{Ct}}$ method.

Biochemical determination of proinflammatory cytokines. The spinal cord tissues were homogenated and the content of IL-1 $\beta$ and TNF $\alpha$ in spinal cord tissue homogenates were measured using ELISA kits according to the manufacturers' instructions (BioVision Inc).

Evaluation of oxidative stress. The content of TBA reactive substances and glutathione (GSH) and superoxide dismutase (SOD) activity were measured using commercial kits according to the manufacturers' instructions (Nanjing Jiancheng, China).

Statistical analysis. The software SPSS 16.0 for Windows (SPSS Inc., Chicago, IL, USA) was used to conduct statistical analyses. The results are presented as mean \pm SEM. The statistical significance of differences between groups was analyzed via one-way analysis of variance followed by a Dunnett's t-test for multiple comparisons. A $P$-value $<0.05$ was considered to be significant.

\section{RESULTS}

\section{Effect of GAS on locomotor function recovery in rats with $\mathrm{SCl}$}

Locomotor function is believed to be a well indicator of injury of spinal cord. In the present study, we evaluated the effect of GAS on recovery of locomotor functions. BBB scores were performed both prior to injury and at $1,3,7,14,21$ and 28 days post-injury. As shown in Fig. 1, rats in the Sham group exhibited a 21-point non-injured score, indicating none injury of locomotor functions of rats in the Sham group. BBB locomotor rating scale was promptly decreased one day after the SCI operation. After that, BBB scores in rats of SCI group slowly increased during 1-28 days, indicating that locomotor functions of rats were gradually recovered. Compared with that of SCI, BBB scores of rats in GAS group were significantly higher during 7-28 days after the operation (Fig. 1), indicating that GAS administration significantly improved the recovery of locomotor function in rats with contusive SCI.

\section{Effect of GAS on permeability of blood-spinal cord barrier in rats with $\mathrm{SCl}$}

It is considered that increased permeability of bloodspinal cord barrier (BSCB) is positively related with SCI (Winkler et al., 2014; Yu et al., 2015). In our study, we

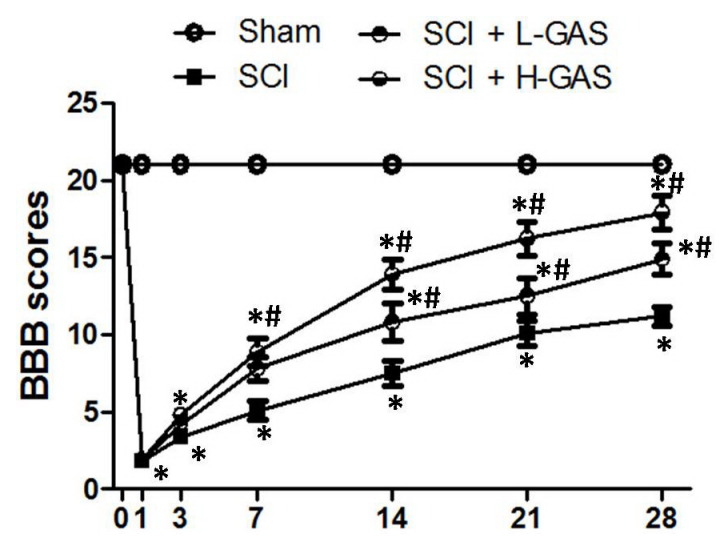

Figure 1. Effect of gastrodin on locomotor function recovery in rats with spinal cord injury.

Prior to surgery and at 1, 3, 7, 14, 21 and 28 days post-injury, the Basso-Beattie-Bresnahan (BBB) scores of rats were observed. ${ }^{*} p<0.05$, compared with Sham group. $\# p<0.05$, compared with $\mathrm{SCl}$ group. Sham: sham-operated group, $\mathrm{SCl}$ : spinal cord injury group, $\mathrm{SCl}+\mathrm{L}-\mathrm{GAS}$ : $\mathrm{SCl}+$ low dose of GAS group; $\mathrm{SCl}+\mathrm{H}-\mathrm{GAS}$, $\mathrm{SCl}+$ high dose of GAS group. 

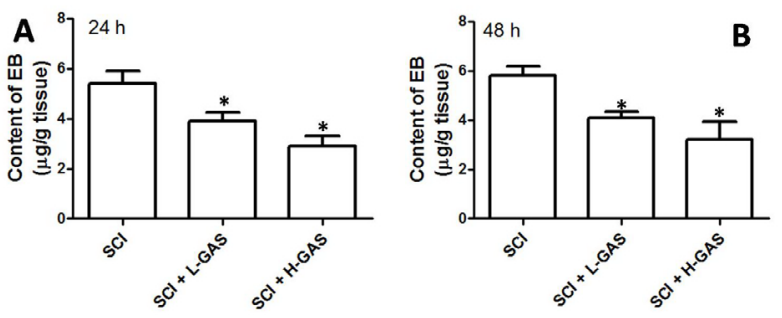

Figure 2. Effect of gastrodin on permeability of blood-spinal cord barrier in rats with spinal cord injury.

The permeability of blood-spinal cord barrier was assessed by Evans blue extravasation $(\mu \mathrm{g} / \mathrm{g}$ spinal cord tissue). (A) mean $\pm \mathrm{SEM}$ : SCl: $5.4 \pm 1.0 ; \mathrm{SCl}+\mathrm{L}-\mathrm{GAS}: 3.8 \pm 0.64$; $\mathrm{SCl}+\mathrm{H}-\mathrm{GAS}: 2.8 \pm 0.79$. (B) mean \pm SEM: SCl: $5.8 \pm 0.73$; SCl+L-GAS: $4.1 \pm 0.45$; SCl+H-GAS: $3.2 \pm 1.21$. ${ }^{*} p<0.05$, compared with $\mathrm{SCl}$ group. SCl: spinal cord injury group, $\mathrm{SCl}+$ L-GAS: $\mathrm{SCl}+$ low dose of GAS group; $\mathrm{SCl}+\mathrm{H}-\mathrm{GAS}, \mathrm{SCl}+$ high dose of GAS group.
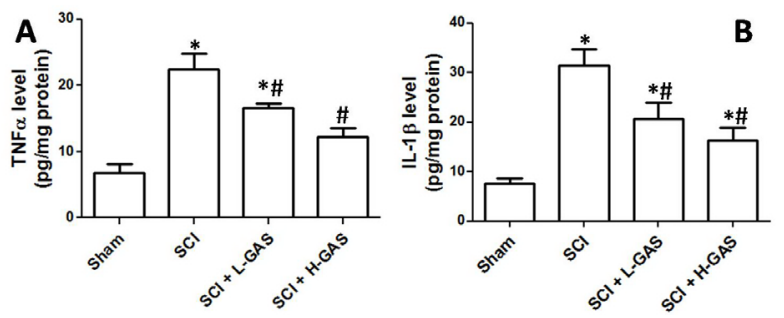

Figure 3. Effect of gastrodin on inflammation in rats with spinal cord injury.

The spinal cord tissues were homogenated and the content of TNFa (A) and IL-1 $\beta$ (B) in spinal cord tissue homogenates were measured using ELISA kits. (A) mean \pm SEM: Sham: $6.8 \pm 3.1$; SCI: 22.4 $\pm 5.4 ; \quad \mathrm{SCl}+\mathrm{L}-\mathrm{GAS}: \quad 16.6 \pm 1.5 ; \quad \mathrm{SCl}+\mathrm{H}-\mathrm{GAS}: 12.2 \pm 2.0$. (B) mean \pm SEM: Sham: $7.6 \pm 2.3$; SCl: $31.4 \pm 2.4$; SCl+L-GAS: $20.6 \pm 6.5$; SCl+HGAS: $16.1 \pm 4.5$. ${ }^{*} p<0.05$, compared with Sham group. $\# p<0.05$, compared with $\mathrm{SCl}$ group. Sham: sham-operated group, SCl: spinal cord injury group, $\mathrm{SCl}+\mathrm{L}-\mathrm{GAS}$ : $\mathrm{SCl}+$ low dose of GAS group; $\mathrm{SCI}$ $+\mathrm{H}-\mathrm{GAS}, \mathrm{SCl}+$ high dose of GAS group.
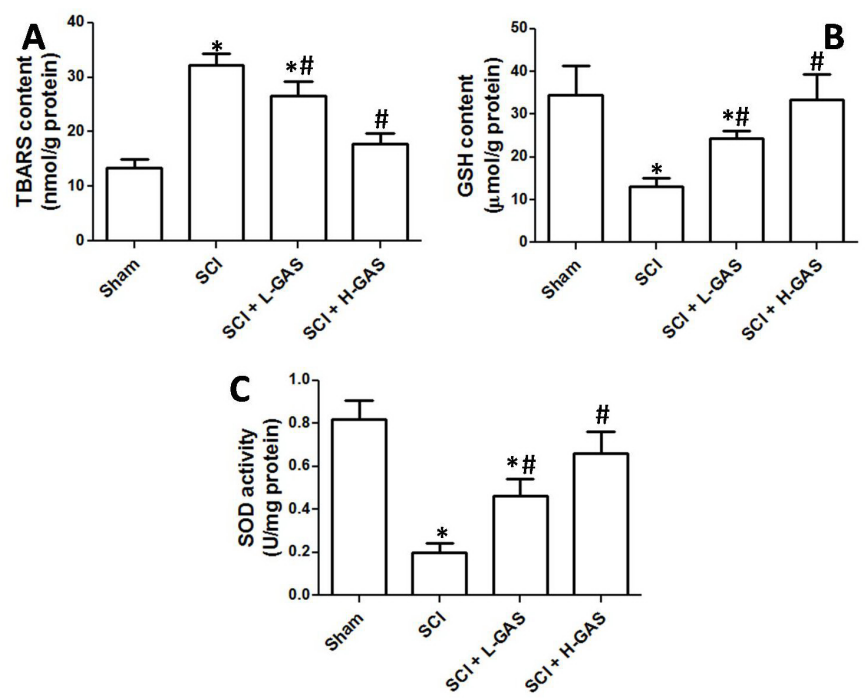

Figure 4. Effect of gastrodin on oxidative stress in rats with spinal cord injury.

The spinal cord tissues were homogenated and TBARS (A) and GSH (B) level and SOD (C) activity were determined by assay kits to assess oxidative stress. (A) mean \pm SEM: Sham: 13.4 \pm 3.6 ; SCl: 32.2 \pm 4.6 ; SCI+L-GAS: 26.6 \pm 5.1 ; SCI+H-GAS: 17.8 \pm 4.3 . (B) mean \pm SEM: Sham: $34.4 \pm 11.3$; SCl: $13.0 \pm 4.5$; SCl+L-GAS: $24.4 \pm 3.8$; SCl+H-GAS: $33.2 \pm 10.5$. (C) mean \pm SEM: Sham: $0.82 \pm 0.12 ; \mathrm{SCl}: 0.2 \pm 0.09 ; \mathrm{SCl}+\mathrm{L}-$ GAS: $0.46 \pm 0.11$; SCl+H-GAS: $0.66 \pm 0.19$. ${ }^{*} p<0.05$, compared with Sham group. $\# p<0.05$, compared with $\mathrm{SCl}$ group. Sham: sham-operated group, SCl: spinal cord injury group, $\mathrm{SCl}+\mathrm{L}-\mathrm{GAS}$ : $\mathrm{SCl}+$ low dose of GAS group; $\mathrm{SCl}+\mathrm{H}-\mathrm{GAS}, \mathrm{SCl}$ + high dose of GAS group. also assessed the effect of GAS on the permeability of BSCB using Evans blue extravasation. The results showed that GAS notably decreased the content of Evans blue in spinal cord tissue compared with that of SCI group (Fig. 2), indicating that GAS administration markedly ameliorated the disruption of BSCB in rats with contusive SCI.

\section{Effect of GAS on inflammation in rats with $\mathrm{SCI}$}

Inflammation is closely associated with development of SCI and anti-inflammatory effect was shown to contribute to improvement of SCI (Allison \& Ditor, 2015; Luo et al., 2015; Lv et al., 2015; Tyagi et al., 2015). In the study, we also evaluated the effect of GAS on inflammation in rats with SCI. As shown in Fig. $3 \mathrm{~A}$ and $\mathrm{B}$, in SCI rats, tumor necrosis factor $\alpha(\mathrm{TNF} \alpha)$ (A) and interleukin$1 \beta$ (IL-1 $\beta$ ) (B) mRNA expression in spinal cord tissue ates were significantly increased compared with that of sham rats. GAS treatment markedly reduced the RNA expression in SCI rats (Fig. 3). The results indicated that the operation of SCI induced significant inflammation and GAS administration exhibited notable anti-inflammatory effect in SCI rats.

\section{Effect of GAS on oxidative stress in rats with $\mathrm{SCl}$}

Increased reactive oxygen species (ROS), also called oxidative stress, is related with the occurrence and development of SCI (Khayrullina et al., 2015; Luo et al., 2015). In the next step, we examined the effect of GAS on oxidative stress status in SCI rats. As shown in Fig. 4A, TBARS content in SCI rats were significantly higher than that of Sham rats and GAS treatment significantly inhibited the hibit lipid peroxidation after SCI. In Fig. $4 \mathrm{~B}$ and $\mathrm{C}$, we wed that glutathione $(\mathrm{GSH})$ content and superoxide dismutase (SOD) activities were significantly decreased in SCI rats. The administration of GAS could remarkably inhibit the decrease of GSH and SOD (Fig. 4B and $\mathrm{C}$ ). These data demonstrated that GAS could effectively ameliorated oxidative stress in rats with SCI.

\section{Effect of GAS on key regulators of oxidative} stress in rats with $\mathrm{SCl}$

To clarify the possible mechanism of GAS-induced protective effects against SCI and reduction of inflammation and oxidative stress, we evaluated the effect of GAS on key regulators of oxidative stress in rats with SCI. The results showed that in rats with SCI, the mRNA expression of nuclear factor (erythroid-derived 2)-like 2 (Nrf2), an important redox controller, was significantly decreased (Fig. 5A). GAS administration significantly inhibited the decrease of $\mathrm{Nrf} 2$ in SCI rats (Fig. 5A). Moreover, mRNA expression of catalytic subunit of $\gamma$-glutamylcysteine ligase (GCLc) and modified subunit of $\gamma$-glutamylcysteine ligase (GCLm), important rate-limiting enzyme for GSH synthesis, was notably reduced by contusive SCI (Fig. $5 \mathrm{~B}$ and $\mathrm{C}$ ). In contrast, GAS administration significantly increased $\mathrm{mRNA}$ expression of GCLc and GCLm in SCI rats. The results demonstrated that the upregulation of $\mathrm{Nrf} 2$, GCLc and GCLm may be involved in the protective effects of GAS in SCI rats. 


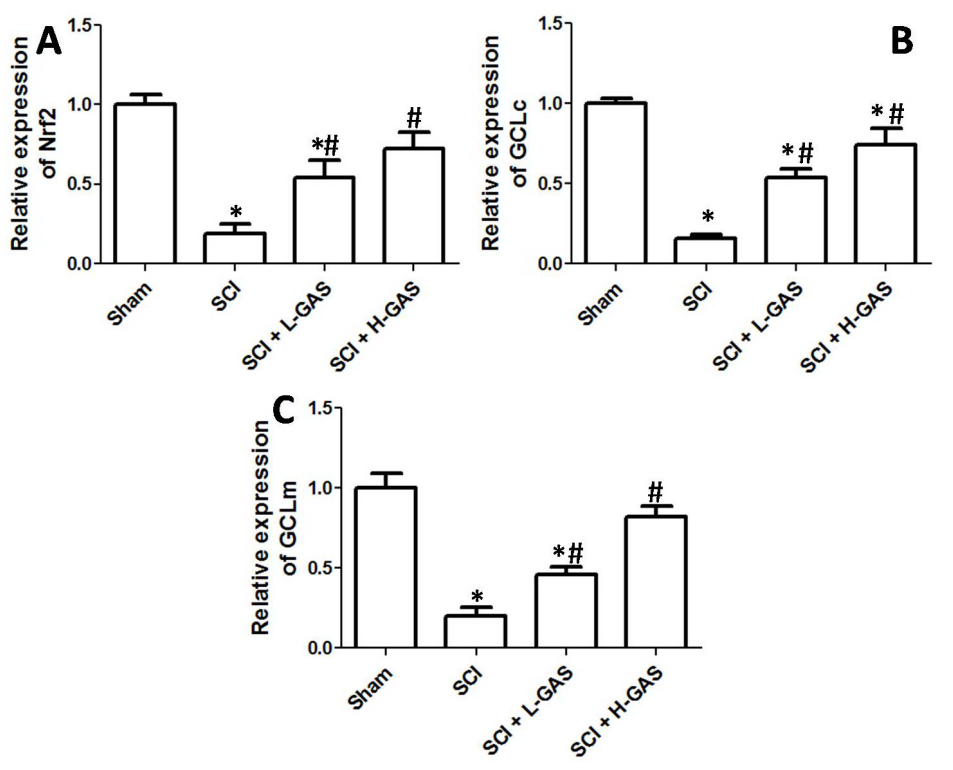

Figure 5. Effect of gastrodin on key regulators of oxidative stress in rats with spinal cord injury.

mRNA expression of Nrf2 (A), GCLc (B) and GCLm (C) was detected by realtime PCR and results were shown as folds of Sham group. (A) mean $\pm S E M$ : Sham: $1.0 \pm 0.12 ; \mathrm{SCl}: 0.19 \pm 0.11 ; \mathrm{SCl}+\mathrm{L}-\mathrm{GAS}: 0.54 \pm 0.22 ; \mathrm{SCl}+\mathrm{H}-\mathrm{GAS}: 0.72 \pm 0.20$. (B) mean \pm SEM: Sham: $1.0 \pm 0.07$; SCl: $0.16 \pm 0.05$; SCI+L-GAS: $0.54 \pm 0.11$; SCI+H-GAS: $0.74 \pm 0.21$. (C) mean \pm SEM: Sham: $1.0 \pm 0.19$; SCI: $0.2 \pm 0.11$; SCI+L-GAS: $0.45 \pm 0.09$; $\mathrm{SCl}+\mathrm{H}-\mathrm{GAS}: 0.82 \pm 0.13$. ${ }^{*} \mathrm{p}<0.05$, compared with Sham group. \#p < 0.05, compared with $\mathrm{SCl}$ group. Sham: sham-operated group, $\mathrm{SCl}$ : spinal cord injury group, $\mathrm{SCl}+\mathrm{L}-\mathrm{GAS}$ : SCI + low dose of GAS group; $\mathrm{SCl}+\mathrm{H}-\mathrm{GAS}, \mathrm{SCl}+$ high dose of GAS group.

\section{DISCUSSION}

Large amount of literature have shown the neuroprotective roles of GAS in vivo and in vitro (Sun et al., 2004; Xu et al., 2007; Dai et al., 2011; Park et al., 2011; Peng et al., 2015). Recently, it was also shown that GAS could improve the recovery of SCI (Song et al., 2013). It is suggested that GAS may both possess neuroprotective activity and facilitate neuro-regeneration. However, the mechanism underlying the effects of GAS on regeneration after SCI is far from completely understood. In the present study, using a model of recovery of SCI, we examined the effect of GAS on inflammation and oxidative stress in rats with SCI and evaluated the possible role of antioxidant and anti-inflammatory activities in GAS-enhanced recovery from SCI.

In the study, locomotor function and permeability of BSCB was determined to evaluate the injury of spinal cord after the operation and GAS administration. Locomotor function is an important indicator of injury of spinal cord in animals (Ewan \& Hagg, 2015). It is suggested that disruption of BSCB is closely associated with increased mortality after endovascular therapy and contributes to early motor-neuron degeneration (Winkler et al., 2014). Improvement of BSCB dysfunction can significantly reduce secondary nerve injury and enhance neuroprotection (Sharma, 2011), implicating that early repair of the BSCB is significant for the treatment of SCI. We showed that GAS could effectively ameliorate SCI, as evidenced by improved recovery of locomotor function and reduced permeability of BSCB.

Inflammation is closely associated with development of SCI and anti-inflammatory effect was shown to con- tribute to improvement of SCI (Allison \& Ditor, 2015; Luo et al., 2015; Lv et al., 2015; Tyagi et al., 2015). IL-1 $\beta$ is elevated following SCI and important for the signaling required for chronic damage (Hayashi et al., 1997; Kleibeuker et al., 2008). Moreover, $\mathrm{TNF} \alpha$ is involved in SCI-related inflammation and downregulation of $\mathrm{TNF} \alpha$ contributes to recovery of SCI (Haan et al., 2015; Yang et al., 2015). We found that GAS could inhibit inflammation in SCI rats, as reflected by decrease of IL- $1 \beta$ and TNF $\alpha$ levels in SCI rats. Previous studies have shown that GAS ameliorated anxiety-like behaviors and inhibited IL- $1 \beta$ level in the rat model of posttraumatic stress disorder (Peng et al., 2013). Li et al showed that GAS inhibited IL-1 $\beta$ and TNF $\alpha$ level in rotenone-induced Parkinson's disease model rats (Li et al., 2012). Combined with these results, it is suggested that anti-inflammatory effect of GAS may be involved in the protective effect of GAS against SCI.

Oxidative stress is related with the occurrence and development of SCI (Khayrullina et al., 2015; Luo et al., 2015). Wang and coworkers (2014) found that GAS prevented motor deficits and oxidative stress in the MPTP mouse model of Parkinson's disease via ERK1/2-Nrf2 signaling pathway. Peng et al. showed that GAS alleviated cerebral ischemic damage in mice by antioxidant and anti-inflammatory activities which involved upregulation of SOD and Nrf2 pathway (Peng et al., 2015). Nrf2/antioxidant response element (ARE) signaling pathway play an important role in the central nervous system (Sandberg et al., 2014; Kieseier \& Wiendl, 2015), defending against potential stress or insult. Wang and coworkers (2014) reported that Nrf2 upregulated ATP binding cassette transporter expression and activity at the blood-brain and BSCB. Nrf2 was found to be the targets of several active substances. It was also found that the effect of ginseng on SCI-induced oxidative stress and inflammatory response was mediated by activation of $\mathrm{Nrf} 2$ (Wang et al., 2015). Activation of Nrf2 was involved in sulforaphane-enhanced recovery after contusive SCI (Benedict et al., 2012). GCLc and GCLm are the ratelimiting enzymes for the synthesis of GSH, an important antioxidant protein in the body. Various studies have suggested that GCLc and GCLm are regulated by $\mathrm{Nrf} 2$ in the defense against oxidative insult (Guan et al., 2015). In the current study, we also tested the effect of GAS on Nrf2, GCLc and GCLm expression. The results showed that GAS significantly increased mRNA expression of Nrf2, GCLc and GCLm in SCI rats, indicating that enhancement of Nrf2-GCLc/ GCLm pathway may be involved in the protective effect of GAS against SCI

In conclusion, the novel finding of our study was that GAS may promote the recovery of SCI through the enhancement of Nrf2-GCLc/GCLm signaling pathway, and then improvement of oxidative stress and inflammation, resulting in decreased permeability of BSCB and improved recovery of locomotor function in SCI rats. These results have provided novel insights into GASrelated therapy of SCI and associated neurodegenerative diseases. 


\section{Conflict of interest}

The authors declare none.

\section{REFERENCES}

Alkabie S, Boileau AJ (2015) The role of therapeutic hypothermia after traumatic spinal cord injury-a systematic review. World Neurosurg 86: 432-449. doi: 10.1016/j.wneu.2015.09.079.

Allison DJ, Ditor DS (2015) Targeting inflammation to influence mood following spinal cord injury: a randomized clinical trial. I Neuroinflammation 1: 204 doi: 10.1186/s12974-015-0425-2.

Basso DM, Beattie MS, Bresnahan JC (1995) A sensitive and reliable locomotor rating scale for open field testing in rats. J Neurotrauma 1 : 1-21. doi:10.1089/neu.1995.12.1.

Benedict AL, Mountney A, Hurtado A, Bryan KE, Schnaar RL, Dinkova-Kostova AT, Talalay P (2012) Neuroprotective effects of sulforaphane after contusive spinal cord injury. I Neurotrauma 16: 2576-2586. doi: 10.1089/neu.2012.2474.

Chen MH, Liu YH, Xu H, Xu DW, Wang CN, Wang Y, Duan CW, Zhou Y, Kan P, Shen AG, Wang YH (2015) Lentiviral vector-mediated p27 expression facilitates recovery after spinal cord injury. Mol Neurobiol. doi: 10.1007/s12035-015-9498-2.

Dai JN, Zong Y, Zhong LM, Li YM, Zhang W, Bian LG, Ai QL, Liu YD, Sun J, Lu D (2011) Gastrodin inhibits expression of inducible NO synthase, cyclooxygenase-2 and proinflammatory cytokines in cultured LPS-stimulated microglia via MAPK pathways. PLoS One 7: e21891. doi: 10.1371/journal.pone.0021891.

Dionyssiotis Y, Mavrogenis A, Trovas G, Skarantavos G, Papathanasiou J, Papagelopoulos P (2014) Bone and soft tissue changes in patients with spinal cord injury and multiple sclerosis. Folia Med (Plovdiv) 4: 237-244. doi: 10.1515/folmed-2015-0002.

Edwards WB, Schnitzer TJ, Troy KL (2014) Bone mineral and stiffness loss at the distal femur and proximal tibia in acute spinal cord injury. Osteoporos Int 3: 1005-1015. doi: 10.1007/s00198-013-2557-5.

Evaniew N, Belley-Cote EP, Fallah N, Noonan V, Rivers CS, Dvorak MF (2015) Methylprednisolone for the treatment of patients with acute spinal cord injuries: A systematic review and meta-analysis. I Neurotrauma. doi: 10.1089 /neu.2015.4192

Ewan EE, Hagg T (2015) Intrathecal acetyl-l-carnitine protects tissue and improves function after a mild contusive spinal cord injury in rats. I Neurotrauma. doi: 10.1089/neu.2015.4030.

Giuliano F, Hultling C, El MW, Smith MD, Osterloh IH, Orr M, Maytom M (1999) Randomized trial of sildenafil for the treatment of erectile dysfunction in spinal cord injury. Sildenafil Study Group. Ann Neurol 1: 15-21.

Guan D, Su Y, Li Y, Wu C, Meng Y, Peng X, Cui Y (2015) Tetramethylpyrazine inhibits $\mathrm{CoCl} 2$-induced neurotoxicity through enhancement of $\mathrm{Nrf2/GCLc/GSH}$ and suppression of HIF1alpha/ NOX2/ROS pathways. J Neurochem 3: 551-565. doi: 10.1111/ jnc.13161.

Haan N, Zhu B, Wang J, Wei X, Song B (2015) Crosstalk between macrophages and astrocytes affects proliferation, reactive phenotype and inflammatory response, suggesting a role during reactive gliosis following spinal cord injury. J Neuroinflammation. 109. doi: 10.1186/ s12974-015-0327-3.

Hayashi M, Ueyama T, Tamaki T, Senba E (1997) Expression of neurotrophin and IL-1 beta mRNAs following spinal cord injury and the effects of methylprednisolone treatment. Kaibogaku Zasshi 3: 209-13.

Henke D, Gorgas D, Doherr MG, Howard J, Forterre F, Vandevelde M (2015) Longitudinal extension of myelomalacia by intramedullary and subdural haemorrhage in a canine model of spinal cord injury. Spine J. doi: 10.1016/j.spinee.2015.09.018.

Hu J, Lang Y, Cao Y, Zhang T, Lu H (2015) The neuroprotective effect of tetramethylpyrazine against contusive spinal cord injury by activating PGC-1alpha in rats. Neurochem Res 7: 1393-1401. doi: 10.1007/s11064-015-1606-1.

Hu JZ, Huang JH, Xiao ZM, Li JH, Li XM, Lu HB (2013) Tetramethylpyrazine accelerates the function recovery of traumatic spinal cord in rat model by attenuating inflammation. I Neurol Sci 1-2: 94-99. doi: 10.1016/j.jns.2012.10.009.

Khayrullina G, Bermudez S, Byrnes KR (2015) Inhibition of NOX2 reduces locomotor impairment, inflammation, and oxidative stress after spinal cord injury. I Neuroinflammation 172. doi: 10.1186/ s12974-015-0391-8.

Kieseier BC, Wiendl H (2015) Nrf2 and beyond: deciphering the mode of action of fumarates in the inflamed central nervous system. Acto Neuropathol 2: 297-298. doi: 10.1007/s00401-015-1457-5.

Kleibeuker W, Gabay E, Kavelaars A, Zijlstra J, Wolf G, Ziv N, Yirmiya R, Shavit Y, Tal M, Heijnen CJ (2008) IL-1 beta signaling is required for mechanical allodynia induced by nerve injury and for the ensuing reduction in spinal cord neuronal GRK2. Brain Behav Immun 2: 200-208. doi:10.1016/j.bbi.2007.07.009.
Li C, Chen X, Zhang N, Song Y, Mu Y (2012) Gastrodin inhibits neuroinflammation in rotenone-induced Parkinson's disease model rats. Neural Regen Res 5: 325-331. doi: 10.3969/j.issn.16735374.2012.05.001

Luo Y, Fu C, Wang Z, Zhang Z, Wang H, Liu Y (2015) Mangiferin attenuates contusive spinal cord injury in rats through the regulation of oxidative stress, inflammation and the $\mathrm{Bcl} 2$ and $\mathrm{Bax}$ pathway. Mol Med Rep 5: 7132-7138. doi: 10.3892/mmr.2015.4274.

Lv R, Mao N, Wu J, Lu C, Ding M, Gu X, Wu Y, Shi Z (2015) Neuroprotective effect of allicin in a rat model of acute spinal cord injury. Life Sci 143: 114-123. doi: 10.1016/j.lfs.2015.11.001.

Park S, Kim DS, Kang S (2011) Gastrodia elata Blume water extracts improve insulin resistance by decreasing body fat in diet-induced obese rats: vanillin and 4-hydroxybenzaldehyde are the bioactive candidates. Eur J Nutr 2: 107-118. doi: 10.1007/s00394-010-0120-0.

Peng Z, Wang H, Zhang R, Chen Y, Xue F, Nie H, Chen Y, Wu D, Wang Y, Wang H, Tan Q (2013) Gastrodin ameliorates anxiety-like behaviors and inhibits IL-1beta level and p38 MAPK phosphorylation of hippocampus in the rat model of posttraumatic stress disorder. Physiol Res 5: 537-545.

Peng Z, Wang S, Chen G, Cai M, Liu R, Deng J, Liu J, Zhang T, Tan Q, Hai C (2015) Gastrodin alleviates cerebral ischemic damage in mice by improving anti-oxidant and anti-inflammation activities and inhibiting apoptosis pathway. Neurochem Res 4: 661-673. doi: 10.1007/s11064-015-1513-5.

Sandberg M, Patil J, D’Angelo B, Weber SG, Mallard C (2014) NRF2regulation in brain health and disease: implication of cerebral inflammation. Neuropharmacology 298-306. doi: 10.1016/j.neuropharm.2013.11.004.

Sharma HS (2011) Early microvascular reactions and blood-spinal cord barrier disruption are instrumental in pathophysiology of spinal cord injury and repair: novel therapeutic strategies including nanowired drug delivery to enhance neuroprotection. J Neural Transm 1: 155176. doi: 10.1007/s00702-010-0514-4.

Song C, Fang S, Lv G, Mei X (2013) Gastrodin promotes the secretion of brain-derived neurotrophic factor in the injured spinal cord. Neural Regen Res 15: 1383-1389. doi: 10.3969/j.issn.16735374.2013.15.005.

Sothmann J, Stander J, Kruger N, Dunn R (2015) Epidemiology of acute spinal cord injuries in the Groote Schuur Hospital Acute Spinal Cord Injury (GSH ASCI) Unit, Cape Town, South Africa, over the past 11 years. S Afr Med J 10: 835-839. doi: 10.7196/SAMJnew.8072.

Sun XF, Wang W, Wang DQ, Du GY (2004) Research progress of neuroprotective mechanisms of Gastrodia elata and its preparation. Zhongguo Zhong Yao Za Zhi 4: 292-295.

Tyagi P, Kadekawa K, Kashyap M, Pore S, Yoshimura N (2015) Spontaneous recovery of reflex voiding following spinal cord injury mediated by anti-inflammatory and neuro-protective factors. Urology 88: 57-65. doi: 10.1016/j.urology.2015.10.017.

Wang W, Shen H, Xie JJ, Ling J, Lu H (2015) Neuroprotective effect of ginseng against spinal cord injury induced oxidative stress and inflammatory responses. Int I Clin Exp Med 3: 3514-3521.

Wang X, Campos CR, Peart JC, Smith LK, Boni JL, Cannon RE, Miller DS (2014) Nrf2 upregulates ATP binding cassette transporter expression and activity at the blood-brain and blood-spinal cord barriers. J Neurosci 25: 8585-8593. doi: 10.1523/JNEUROSCI.2935-13.2014.

Wang XL, Xing GH, Hong B, Li XM, Zou Y, Zhang XJ, Dong MX (2014) Gastrodin prevents motor deficits and oxidative stress in the MPTP mouse model of Parkinson's disease: involvement of ERK1/2-Nrf2 signaling pathway. Life Sci 2: 77-85. doi: 10.1016/j. Ifs.2014.08.004.

Winkler EA, Sengillo JD, Sagare AP, Zhao Z, Ma Q, Zuniga E, Wang Y, Zhong Z, Sullivan JS, Griffin JH, Cleveland DW, Zlokovic BV (2014) Blood-spinal cord barrier disruption contributes to early motor-neuron degeneration in ALS-model mice. Proc Natl Acad Sci USA 11: E1035-42. doi: 10.1073/pnas.1401595111.

Xu X, Lu Y, Bie X (2007) Protective effects of gastrodin on hypoxiainduced toxicity in primary cultures of rat cortical neurons. Planta Med 7: 650-654. doi: 10.1055/s-2007-981523.

Yang T, Wu L, Wang H, Fang J, Yao N, Xu Y (2015) Inflammation level after decompression surgery for a rat model of chronic severe spinal cord compression and effects on ischemia-reperfusion injury. Neurol Med Chir (Tokyo) 7: 578-586. doi: 10.2176/nmc.oa.2015-0022.

Yu DS, Cao Y, Mei XF, Wang YF, Fan ZK, Wang YS, Lv G (2014) Curcumin improves the integrity of blood-spinal cord barrier after compressive spinal cord injury in rats. J Neurol Sci 1-2: 51-59. doi: 10.1016/j.jns.2014.07.056

Yu DS, Liu LB, Cao Y, Wang YS, Bi YL, Wei ZJ, Tong SM, Lv G, Mei XF (2015) Combining bone marrow stromal cells with green tea polyphenols attenuates the blood-spinal cord barrier permeability in rats with compression spinal cord injury. I Mol Neurosci 2: 388-396. doi: 10.1007/s12031-015-0564-z. 